\title{
Guang Yuan Low-carbon Development Path Based on SWOT Analysis
}

\author{
Xiaoqin $\operatorname{Jin}^{1}$ \\ ${ }^{1}$ Department of Economic Management, Sichuan Agricultural University, Sichuan, China \\ Correspondence: Xiaoqin Jin, Department of Economic Management, Sichuan Agricultural University, Dongbei \\ road 555, Wenjiang, Chengdu 611130, SiChuan, China. Tel: 86-138-8001-5631. E-mail: jxq03@163.com.cn
}

Received: May 30, 2012 Accepted: July 22, 2012 Online Published: July 25, 2012

doi:10.5539/jsd.v5n8p78 URL: http://dx.doi.org/10.5539/jsd.v5n8p78

This research is financed by Chinese National Social Science; the project is: To cope with climate change under the urban ecological environment sustainable development and the construction of ecological civilization in China (09\&ZD049)

\begin{abstract}
The low-carbon economic model is based on low energy consumption, low pollution, low emission; it is another major advancement of human society following agricultural civilization and industrial civilization. Low-carbon economy with its unique advantages and huge market has become a hot spot of world economic development. Now western region in China is facing two major tasks of the leapfrog development and sustainable development, as the less developed areas and the Wenchuan quake-hit, Guang Yuan has its representation and specificity. To achieve low-carbon reconstruction and sustainable development, how to choose a low-carbon path is very important for Guang Yuan. In this paper, it analyzed the advantage, weaknesses, opportunities and threats by SWOT method, and then proposed a feasible path for Guang Yuan's low-carbon development; it also hoped to provide a reference for the scientific development of underdeveloped areas.
\end{abstract}

Keywords: low-carbon economy, SWOT analysis, Guang Yuan city, development path

\section{Introduction}

The low-carbon economic model is based on low energy consumption, low pollution, low-emission; it is another major advancement of human society following agricultural civilization and industrial civilization. Low-carbon economy with its unique advantages and huge market has become a hot spot of world economic development. At present, western region in China is in the critical period of industrialization and urbanization, it is undergoing domestic and international industrial transfer test, facing two major tasks of the leapfrog development and sustainable development. During the 12th Five-Year Plan period in China, under the background of new round western big development opportunity, western region must transform the mode of development and switch to low carbon development path, so as to alleviate the contradiction between economic growth and environment. As the less developed areas and the Wenchuan quake-hit, Guang Yuan has its representation and specificity. Therefore, it is worthy of study to using SWOT method to analysis Guang Yuan's low-carbon Development path.

\section{General Situation in the Study Area}

Guang Yuan city is located at $104^{\circ} 36^{\prime} \sim 106^{\circ} 45^{\prime} \mathrm{E}$, latitude $31^{\circ} 31^{\prime} \sim 32^{\circ} 57^{\prime} \mathrm{N}$, the PenZhou mountains to the north of Sichuan, the Jialing River upstream. It is the north gate of Sichuan. Guang Yuan city was established in 1985, covering three states of Lizhou, Yuanba, Chaotian and four counties of Cangxi, Wangcang, Jiange and Qingchuan, with a vast area of 1.63 square kilometers, the total population of 3.03 million, and the urban population 480000. In 2011, the gross domestic product (GDP) was to 40.354 billion Yuan, increased by 15.6 percent. Value added of large-scale industry reached 13.87 billion Yuan, increased by 30.4 percent, ranking first in Sichuan province.

Guang Yuan's low-carbon development is put forward from November 2008. On May 12th, 2008 Wenchuan earthquake occurred, it made Guang Yuan became the earthquake-stricken area, so Guang Yuan hosted a forum for its reconstruction. Experts from 47 universities of China and Chinese Academy of Social Sciences and Dr. group sent from the central mission gave advice and suggestions for Guang Yuan's reconstruction. With full 
respect for expert advice and based on Guang Yuan's own advantages, Guang Yuan decided to choose the low-carbon path to the recovery. After three years of hard work, Guang Yuan's low-carbon reconstruction and development have achieved good results. By the end of 2011, it has successfully applied for soil testing and fertilizer emission reduction projects, cooperated to launch low-carbon transportation card by Shanghai World Expo and Guangzhou Asian Games, successfully participated in 5 voluntary emissions trading projects, the transactions is amounting to $\$ 175$ million in total. It has received "Low-carbon Chinese contribution city", and "Low-carbon development outstanding contribution city", and "Low-carbon ecological advanced city" and "Qutstanding green ecological city" titles. In 2011, Guang Yuan is the only city of China's western region which was invited to participate in the UN climate conference in Durban; this is also the affirmation of the United Nations for Guang Yuan's low-carbon development.

\section{Method}

SWOT analysis method, is also called the situation analysis, and respectively represents: Strength, Weakness, Opportunity and Threat. From the whole, SWOT can be divided into two parts: the first part is SW mainly used to analysis the internal conditions; the second part is OT, mainly used to analyze the external environment. Therefore, according to the internal and external actual environment, this paper is to use the SWOT method to analysis the development trend of Guang Yuan's low-carbon economy.

\subsection{Opportunity and Threat}

From the international perspective, especially in the energy crisis and climate change background, the economic development of the rigid constraints undergoing gradually, global will change to a low-carbon economy. From domestic look, in August 2010, the national development and reform commission in China determined to launch a pilot project on low-carbon provinces and low-carbon cities, and take Guangdong, Liaoning, Hubei, Shanxi, Yunnan And Tianjin, Chongqing, Shenzhen, Xiamen, Hangzhou, Nanchang, Guiyang, Baoding etc. as the experimental unit, it marked China's economy stepping on low-carbon road.

Right now, Guang Yuan is in multiple tasks of industrialization, urbanization and recovery period, as an important ecological defense and the sources of rivers, Guang Yuan's economic development will face more and more stringent environmental constraints. At the same time, as the underdeveloped area, Guang Yuan has the opportunity to leapfrogging development with its advantage of backwardness. It may turn to more efficient low-carbon industry structure, and directly into the low-carbon economy development stage. Therefore, from the international and domestic environment, to choose a low-carbon economy development path is not only challenge but also the new opportunity for Guang Yuan.

\subsection{Strength}

\subsubsection{Location Advantages}

Guang Yuan is located in the conjunction of Sichuan, Gansu And Ningxia provinces, it is the green ecological barriers and the upper reaches of the Jialing River. Since ancient times, Guang Yuan has been the transportation hub and material distribution center of Sichuan. During the 12th Five-Year Plan period in China, Guang Yuan has initially established to focus on building regional subprime comprehensive transportation hub, now it is speeding up the construction of the regional commerce, logistics, and tourism center, striving to build an economic and cultural ecological strong city, and it is accelerating to the construction of integrated traffic hub of northwest China and southwest. Therefore, the location advantages become more and more obvious.

\subsubsection{Resource Advantages}

Within the jurisdiction, Guang Yuan is rich in coal, natural gas and hydropower resources. Just now, the coal reserve is to 464 million tons; water reserves is to 2.96 million $\mathrm{kW}$, natural gas reserves is to 400 billion cubic meters. It is the few province of the energy output areas in China. After efforts, Guang Yuan has initially established a new low-carbon energy system. In 2011, clean energy accounted for 23.36 percent of the city's total primary energy consumption, increased by 3.36 percent point from that of the previous year; but the world average level is 35.75 percent, national average is only 9.88 percent, Guang Yuan is higher than the national average. Rural biogas penetration has reached 47 percent; natural gas reached 75 percent in the city. The forest coverage rate has reached 53.6 percent, ranking second in Sichuan province; the forest carbon advantage is obvious.

\subsubsection{The Policy Advantages}

Since 2008, Guang Yuan introduced the policies about "Energy consumption per unit of GDP index statistics and monitoring procedures", "Main pollutant total emission evaluation method" and "Target responsibility 
evaluation method of energy conservation", which reflect a low-carbon economy requirements of the policy, and prepared for the reconstruction of low-carbon economy development. In order to promote low-carbon development, the city government of Guang Yuan has promulgated series guidance documents about "Promoting clean energy and achieving low-carbon development in the construction of circular economy industry park", "The clean energy development and utilization plan", "The circular economy industry park development planning". In 2011, Guang Yuan formulated low-carbon economy development planning for the 12th Five-Year Plan period, which provides policy guarantee for the further development of low-carbon in future.

\subsubsection{The Backwardnes Advantages}

The advantage of backwardnes is the inherent, objective of favorable conditions, which comparison with advanced regions. Guang Yuan belongs to the less developed regions, it is in the early stage to accelerate industrialization and urbanization towards the middle, economic model and the industrial system has not yet been fully designed, "carbon lock" effect is relatively small. If turn to low-carbon economy, Guang Yuan has advantages of backwardness such as smaller resistance, quicker action and lower cost.

\subsubsection{The Foundation of the Low-Carbon Development Advantages}

(1) Achievements in energy saving and emission reduction is remarkable. In 2010, energy consumption per unit GDP was 1.238 tons of standard coal per ten thousand Yuan, decreased by 6.12 percent from that of 2010, accumulatively decreased by 20.11 percent in the 11th Five-Year Plan period; in 2011, energy consumption per unit GDP was 0.984 tons of standard coal per ten thousand Yuan, decreased by 3.22 percent from the previous year. The unit energy consumption has been falling; it laid a solid foundation for Guang Yuan's development of low-carbon economy.

(2) Low-carbon industry has taken shape. Guang Yuan currently has formed the industrial system to certain scale, which mainly about energy, metals, agricultural and sideline products, building materials, electronics, machinery pharmaceutical and chemical industry, etc. Now, low-carbon industry accounts for more than 40 percent of the total industry. Low-carbon agriculture has big development, which with "green agriculture, zoology agriculture and circulating agriculture" as the main contents, low pollution industry such as tourism and service and so on has potential development.

(3) Low-carbon concept is rooted in the heart of everyone. In Guang Yuan, August 27th of each year has been identified as "low-carbon day", every Wednesday as "green travel day" since March 2012. Through variety theme of low-carbon activities, low-carbon concept is rooted in the heart of everyone; the atmosphere of low-carbon development is more and more strong.

\subsection{Weakness}

\subsubsection{The Low-Carbon Industry System Is to Be Improved}

Although the foundation of Guang Yuan's low-carbon industry development is better, a lot of projects are still in a demonstration stage at present, and it also has some contradictions and restricting factors in the process. By 2011, the three industrial structures have been adjusted to 20.8: 44.6: 34.6, the traditional industrial raw materials are still mainly in the overall industrial development, heavy industry proportion to 70percent, modern service sector is less than 40percent, and the service industry is declining consistently. Low-carbon development is in a "point" shape, urban and rural low-carbon economy development is not balanced, it is lack of sufficient fusion between industry, outstanding performance in the industry and the third industry, agriculture and service industry cannot contact closely, low-carbon industry chain effect has not been revealed, and industrial structure adjustment is difficult. Therefore, in order to construct a low-carbon industry system is still on a long road.

\subsubsection{The Energy Structure Is not Reasonable}

At present, the city's energy production mainly concentrated in the coal industry, power industry is forming, and new energy and natural gas industry has just started. Coal, oil are still a leading position on energy consumption. The coal consumption has reached 3.32 million tons (folded by 2.3715 million tons of standard coal), only 2780 small boiler raw coal consumption is more than 300000 t each year, 380000 tons product oil consumption, amounting to 452900 tons of standard coal, accounting for 13.6 percent of the total energy consumption in Guang Yuan; natural gas consumption has reached 105 million cubic meters, or 139700 tons of standard coal, accounting for 3.51 percent of the total energy consumption.

\subsubsection{The Situation of Energy Saving and Emission Reduction is Grim}

Rightly, energy conservation and emission reduction in energy saving can be done from structure, technology and management. Because a number of high load project has fully completed and put into production, such as 
conch cement, high strength cement, guangwang cement and so on, the industrial energy consumption is on the rise, it promoted the energy consumption of value added industry which scale is above ten thousand Yuan rapidly rising, and so brought an unprecedented difficulty and pressure energy conservation and consumption reduction to industry. The task of energy-saving is still heavier, not only in structure but also in technology and management, plus county interval energy cognition, and the imbalance on energy saving job, in order to achieving the annual goals of cutting 3.2 percent energy consumption per unit of GDP and completing the target unit GDP energy consumption decrease 15 percent which issued by Sichuan Province during the 12th Five-Year Plan period, the situation is still grim.

\subsubsection{The Low-Carbon Development Ability Is Insufficient in Backup}

At present, Guang Yuan's low-carbon construction in talent, technology, capital, information and other aspects of the foundation is relatively weak; the low-carbon development ability is insufficient in backup. Low-carbon technology is the important support, low-carbon of professional personnel is the important safeguard in "Low-carbon Guang Yuan", but lack of funding is the key. As the less developed area, Guang Yuan's weak economic foundation, limited financial resources, especially in the post-disaster reconstruction after investment is finished, Guang Yuan may become investment depressions, insufficient funds will be an obstacle in Guang Yuan's sustainable Low-carbon development.

\section{Solutions}

\subsection{Guang Yuan's Low-carbon Construction Should Be Promoted Gradually from Point to Area}

Currently, in order to win the support of the country, Guang Yuan is trying to declare national low-carbon pilot city, and actively striving for more national low-carbon projects. Therefore, Guang Yuan's low-carbon construction should take low-carbon pilot demonstration city as the breakthrough point, and build energy efficiency, low-carbon community construction, low-carbon transportation, etc. for the full implementation of the low-carbonization city management. On one hand, Guang Yuan should choose a batch of better foundation and representatiion of the village (community), and enterprises, institutions and schools as a pilot demonstration unit, and cultivate a group of advanced type covering different areas and different levels, different areas and different industry development of low-carbon. On the other hand, through "Energy conservation and emission reduction model communities", "Low-carbon family" and "Low-carbon civilization traffic family" and "Low-carbon life innovation star", "Low -carbon development outstanding contribution unit" and the form of recognition, mobilize the whole city's initiative and enthusiasm to participate in low-carbon construction. Through the typical demonstration, from point to area, Guang Yuan's low-carbon construction should be promoted gradually.

\subsection{Guang Yuan Should Adjust the Industrial Structure and Build a Low-carbon Industrial System}

Firstly, to develop green agriculture as the main direction, continue to promote soil testing technology and other new technology formula so as to reduce pollution. Vigorously promote ecological agriculture and agricultural circulation economy development, extend and improve "pig-marshy-food", "the pig-marshy-fruit" ecological agriculture circulation patterns and "orchard + farm" leisure tourism mode. Secondly, firmly establish the concept of low-carbon project priority to the development in industry, vigorously promote electronic machinery, agricultural product processing, clean energy and energy conservation and environmental protection industries development, based on saving energy and reducing consumption, based on recycling, based on the traditional industry links transformation, trying to introduce low-carbon enterprise with high technology, low energy consumption and low pollution, low emission and recycling established in Guang Yuan, strive to make new energy and environmental protection industry value scale above account for 60 percent in the large-scale industry, and ten thousand Yuan of large industrial energy consumption is in the lower level.

Thirdly, to improve the proportion of modern service industry, the proportion of the service industry in developed countries are relatively high, generally can reach 70-80 percent, but Guang Yuan is less than 40 percent, so the future development of the space is relatively large. Especially the tourism industry has great potential in development of low pollution. Guang Yuan should rely on its cultural resources such as Shu culture, the queen culture, the red army culture, the folk culture and so on, and foster Guang Yuan city cultural image with characteristics.

\subsection{Guang Yuan should Optimize Energy Structure and Realize Energy Saving and Emission Reduction}

On one hand, combined with Guang Yuan's energy advantages, we should vigorously develop green energy and utilization such as water power, solar, geothermal, biomass energy and so on, speed up the "Guang Yuan gasification" process, and improve the energy structure. On the other hand, government, schools, hospitals and 
other public institutions is to focus on the energy saving transformation, continue to promote energy management contract (EMC), and encourage stores, restaurants and other public institutions building energy management contract by the implementation of energy-saving.

\subsection{Guang Yuan Should Develop the Carbon Potential and Promote Biological Soliding Carbon}

The development of low-carbon economy means not only "to reduce the carbon", but also "to increase carbon sink". Forest plays an irreplaceable role in carbon. According to the concerned data, 1 cubic meters of forest reserves is to absorb carbon dioxide 1.83 tons every year, to release 1.62 tons oxygen. Based on Guang Yuan's forest resources advantage, according to the ecological construction and WenChuan earthquake zone reconstruction planning in SiChuan province, with the opportunity to create "National forest city", Guang Yuan should continue to increase biological solid carbon through the strengthening of returning farmland to forest, farmland conversion, recovery of city green space construction, wetland conservation and restoration.

\subsection{Guang Yuan Should Improve Low-Carbon Ability and Form Long-term Mechanism}

\subsubsection{Paying Attention to Low-Carbon Technology Research and the Construction of Talent Team}

Guang Yuan has established a good relationship with many domestic and foreign universities and research institutes in low-carbon reconstruction, it should continue to speed up the innovation system that the enterprise as the main body, the market as the guidance, the combination of production and technology, and to strengthen local enterprises' independent innovation ability and the core competitive power. By supporting the university and the enterprise constructing training base together, through the "please come in" and "send out" means, nurturing local management, manufacturing and technology talents in low-carbon field. Meanwhile, in view of the current difficult in independent innovation, Guang Yuan should strengthen with other brother city in low-carbon technical cooperation, and focuses on the existing low-carbon technology integration, by increasing the financial subsidies policy measures to speed up the popularization and application of existing low-carbon technology. In addition, by setting up "Year low-carbon innovation contest", further build the environment of respecting talented person and knowledge, so as to incentive the whole society to participate in low-carbon construction.

\subsubsection{Increasing Capital Investment and Perfectting the Financing Mechanism}

As an under developed area, Guang Yuan's economic foundation is weak, the development of low-carbon economy and financial resources is limited, and so it is necessary to build diversified, multi-channel investment and financing system. On one hand, through the integration of existing energy saving capital and science and technology special fund, Guang Yuan should establish low-carbon fund and strive for low-carbon demonstration area construction into the major national science and technology development strategy so as to establish key technology R \& D funds. On the other hand, expand the channel of foreign capital; actively make use of World Bank, ADB, the global environment fund, United UNDP and other international organizations and government's loans or grants. It is worth mentioning that, the state of main function region ecological compensation mechanism will been gradually built and implemented, this also infuse vigor for Guang Yuan's low-carbon development.

\subsection{Guang Yuan Should Cultivate Carbon Trading Market and Strengthen External Cooperation}

On April 12, 2011, Guang Yuan Environmental Exchange official was formally approved. Up to now, it has successfully implemented "The Sino-US cooperation in agriculture greenhouse gas emission reduction "projects and trade," The green travel public transit card", "Asian Games green travel low-carbon card" carbon neutral trading program, realized the interaction of ecological, economic and social benefits. Guang Yuan Environmental Exchange, as the first environmental equity trading mechanism in Sichuan Province, the future development should be based on Sichuan, and strive to build into the western region of carbon trading platform. Guang Yuan has put forward to build "China's first professional carbon index supply city" strategic goal, the sale of carbon emission reduction targets will become new economic point of growth in Guang Yuan's low-carbon development.

\subsection{Guang Yuan Should Strengthen the Propaganda and Advocate Low-carbon Life Consumption}

To achieve the transformation of low-carbon, the concept and behavior of people must be changed, through all kinds of measures to guide people form low-carbon pattern of life and consumption such as publicity, education etc. Firstly, promote green government affairs. According to the government purchasing guidelines and requirements, it is prior to purchase green products. Secondly, improve information sharing platform and implement paperless office. Thirdly, we need to reduce the living energy consumption. On one hand, encourage a housing energy-saving decoration; on the other hand, continue to do the transformation of building energy 
efficiency. Lastly, we need to reduce transportation energy consumption. Encourage people to establish green traffic idea, try to select the public transportation, and advocate "the ecological driving". In addition, encourage economizing, against "Face consumption"; "Luxury consumption" is also very important to reduce energy consumption.

\section{Conclusion}

In short, low carbon economy is not a fashionable concept; it must be put into reality. From macroscopic perspective, we need government leadership, including institutional innovation, perfect market competition mechanism, technological innovation, energy-saving emission reduction as well as the development of low carbon economy policy, actively carry out international cooperation, so as to guide and promote the development of low carbon economy. From the meso level, it is important to carry on the adjustment of industrial structure and vigorously develop strategic emerging industries. From the macroscopic angle, we need enterprises and public awareness, not only changing the traditional consumption concept, but also advocating low carbon life style and mode of production, so as to promote the development of low-carbon economy "collective action". As the less developed areas, Guan Yuan's practice of low-carbon development path has begun to attract wide attention both at home and abroad. Guan Yuan's low-carbon development path is to be sure and the similar area can draw lessons from it.

\section{References}

Du, Sh. F. (2009). Environmental Economics. China Encyclopedia Press, 2009.

Liu, Sh. Y., \& Xu, L. D. (2010). Low-carbon economy discussion in underdeveloped area. Modern Mall, 2010(36).

Jiang, W. (2009). Low-carbon reconstruction-earthquake disaster areas of Guang Yuan's strategic choices. Open Report, 2009(10).

Wang, Zh. F. (2011). Relative advantages and mission analysis to develop low-carbon economy in underdeveloped area. Development Research, 2011(5).

Yu, Y. G. (2011). Strategic path in low-carbon city construction: three type city construction in Ning Bo. Journal of the Southwest National University, 2011(2).

Zhao, M. (2010). Analysis on the feasibility to develop low-carbon economy in western region. Theory DaoKan, 2010(9). 